\title{
An asthmatic child with allergic bronchopulmonary aspergillosis (ABPA)
}

\section{Öner Özdemir}

Division of Allergy and Immunology, Department of Pediatrics, Research and Training Hospital of Sakarya University, Adapazarı, Sakarya, Turkey.E-mail: ozdemir_oner@hotmail.com

Received: 19th July 2017, Revised: 17th August 2017, Accepted: 30th August 2017

SUMMARY: Özdemir Ö. An asthmatic child with allergic bronchopulmonary aspergillosis (ABPA). Turk J Pediatr 2018; 60: 446-452.

Allergic bronchopulmonary aspergillosis (ABPA) results from a TH2 hypersensitivity lung disease in response to bronchial colonization of Aspergillus fumigatus in the respiratory tract. ABPA is a very rare disease in the pediatric population; the prevalence is anticipated to be about $1-2 \%$ in adult asthma patients and $2-15 \%$ in cystic fibrosis patients. In pediatric asthma patients, only rare case reports on ABPA have been published. We present a case of a 12-year-old boy admitted to our hospital with worsening (mild persistent) asthma symptoms such as a recurrent cough, episodic wheezing, and dyspnea for the last two years. He was diagnosed with pneumonia several times and suspected of having tuberculosis during this time. Chest $\mathrm{X}$-ray showed bilaterally perihilar opacities and computed tomography findings revealed central bronchiectasis. With the clinical and laboratory findings, the patient fulfilled 7/8 major diagnostic criteria for ABPA. Treatment with oral corticosteroids and itraconazole was effective. To the best of our knowledge, this is the first reported case of pediatric ABPA with previously documented asthma from Turkey.

Key words: allergic bronchopulmonary aspergillosis, asthma, central bronchiectasis, aspergillus fumigatus.

Allergic bronchopulmonary aspergillosis (ABPA) is a T-helper $2\left(\mathrm{TH}_{2}\right)$ hypersensitivity reaction to fungus, known as Aspergillus fumigatus (AF), allergens colonizing the bronchial tree. ${ }^{1,2}$ Although ABPA is infrequently seen in children, it is more common in children with cystic fibrosis (CF). ${ }^{3,4}$ ABPA is predominantly seen in adult asthmatics, especially corticosteroid dependent asthmatics, in the third and fourth decades of life. Its prevalence varies from $1 \%$ to $25 \%$ of persistent asthma patients in Europe. ${ }^{5-7}$ In 1985 , pediatric persistent asthma patients were evaluated for ABPA by Chetty et al. ${ }^{8}$ and the prevalence was demonstrated to be $15 \%$. There are also probably significant regional differences in ABPA prevalence, with a lower rate likely in the USA and higher rate likely in India. ${ }^{7,9}$ Furthermore, Denning et al. ${ }^{10}$ have estimated that the global prevalence of ABPA may be $0.7-3.5 \%$ of patients with asthma.

The pathogenesis of ABPA is not fully known. Several type of immune responses seem to be implicated, including AF-specific IgEmediated type I, specific IgG-mediated type III hypersensitivity reactions, and abnormal T-lymphocyte responses. ${ }^{11}$ There are some genetic mutations like single-nucleotide polymorphisms and expression of some human leukocyte antigen (HLA) blamed for ABPA susceptibility. Chauhan et al. ${ }^{12}$ reported that asthmatic patients expressing HLA-DR2 and/ or DR5 and possibly HLA-DR4 or DR7 and lacking HLA-DQ2 were at amplified risk to have ABPA after exposure to AF. ${ }^{3}$

ABPA diagnosis might be easily overlooked or delayed, because its clinical picture is often impossible to differentiate from those of

Presented at the 23 ${ }^{\text {rd }}$ National Congress of the Turkish National Allergy and Clinical Immunology Association, 8-12 October 2016, Bodrum, Muğla, and 60th Turkey National Pediatrics Congress, 09-13 November 2016, Antalya and Pediatric Allergy and Asthma Academy Association, 12th Congress, 23-26 April 2017, Kuşadası, Aydın, Turkey 
more frequent lung disorders e.g. uncontrolled asthma, pneumonia and tuberculosis. ${ }^{13}$ ABPA is distinguished by exacerbations of asthma, worsening of pulmonary function, recurrent transient or fixed pulmonary infiltrates, peripheral blood and pulmonary eosinophilia and elevated total IgE level. ${ }^{3}$ Here, we report a 12-year-old boy who was investigated for recurrent cough, pneumonia, episodic wheezing (worsening mild persistent asthma symptoms) and later diagnosed as ABPA with underlying asthma.

\section{Case Report}

A 12-year-old boy [height: $1.38 \mathrm{~cm}$ (3-10p), weight: $28 \mathrm{~kg}(<3 \mathrm{p})$ ] was referred to our department due to worsening asthma symptoms (e.g. intractable cough, episodic wheezing and dyspnea) for two years and suspicion of tuberculosis. In his past medical history, he was previously treated for bronchopneumonia a couple of times and montelukast, inhaled corticosteroids (fluticasone inhaler, $125 \mu \mathrm{g}$, bid) and salbutamol were prescribed for mild persistent asthma since he had been diagnosed with asthma at 9 years of age. Although his cough and symptoms regressed somewhat with the treatment, he did not improve entirely. While he was investigated for uncontrolled asthma symptoms, bilateral perihilar opacities (Fig. 1a) on chest X ray (CXR) and central bronchiectasis on his computed tomography (CT) (Fig. 2a-c) were detected. In his family history, nothing was significant.

During the course of admission, respiratory rate was 20 per minute; oxygen saturation measured by pulse oxymetry was $96 \%$ and body temperature was $37^{\circ} \mathrm{C}$. The patient appeared to be healthy with no signs of respiratory distress. Auscultation of the chest revealed no ronchi or crackles. Further physical examination demonstrated no other pathological findings. Complete blood cell count showed white blood cells: $8.500 / \mathrm{mm}^{3}$ (neutrophils, 30.6\%; lymphocytes, $42.2 \%$;

Table I. Comparison of Laboratory and Clinical Findings of Our Patient with Rosenberg-Patterson Criteria.

Rosenberg-Patterson Criteria Our case (the patient)

Major criteria ${ }^{1,2}$

1. Asthma (spirometric) deterioration not attributable to another etiology

2. Skin prick test to Aspergillus fumigatus

3. Elevated serum specific IgG and IgE to Aspergillus fumigatus Specific IgE greater than twice non-ABPA IgE A. fumigatuspositive asthmatics

4. Serum precipitating IgG antibodies (precipitins) to Aspergillus fumigatus

5. Elevated total serum IgE level $\geq 1000$ (or $\geq 417$ ) IU $/ \mathrm{ml}$

6. Peripheral blood eosinophilia ( $\geq 1.000$ cells $/ \mu \mathrm{L}$ )

7. Chest radiographic transient or fixed lung infiltrate(s) (fleeting shadows)

8. Lung CT: Central (proximal) bronchiectasis in the upper lobes

Minor criteria

10. Delayed type (type III, Arthus type) skin reaction to Asp. antigens

11. Presence of Aspergillus in sputum

12. Golden (brownish black) sputum /mucus plaques
1-) FEV1:63\%; FVC:52\%

2-) FEV1:83\%; FVC:71\% (reversibility+)

Positive

$135 \mathrm{mg} / \mathrm{L} / 38.6 \mathrm{KU} / \mathrm{L}$

N/A

$1.986 \mathrm{IU} / \mathrm{ml}$

$\% 20.2\left(1.720 / \mathrm{mm}^{3}\right)$

Positive

Positive

N/A

Negative

Positive

(1) Criteria 1-8, ABPA-central bronchiectasis, ABPA-CB; (2) Criteria 1-7, ABPA-seropositive, ABPA-S. ABPA: Allergic bronchopulmonary aspergillosis, $\mathrm{N} / \mathrm{A}=$ not available, FEV1 = forced expiratory volume in 1 second, $\mathrm{FVC}=$ forced vital capacity. 

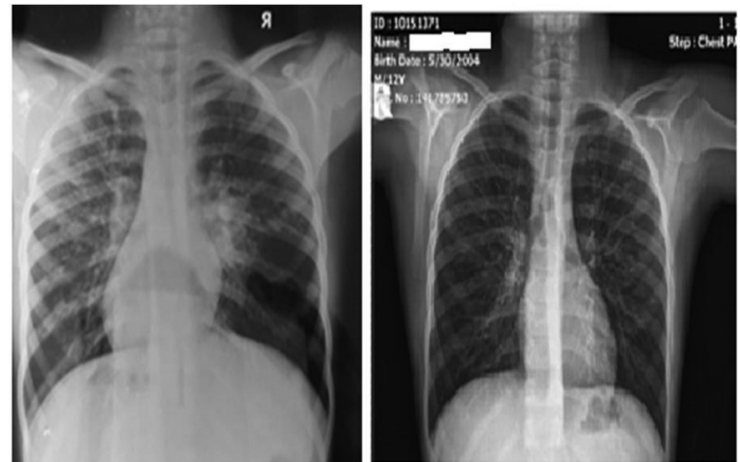

Fig. 1a. Chest radiograph on admission showing perihilar consolidations in the right and left lobes. b. Chest radiograph after 2 months of combined steroid and itraconazol therapy.

monocytes, $6.18 \%$; eosinophils, $20.2 \%$ (1.720/ $\mathrm{mm}^{3}$ ); basophils, $0.80 \%$ ); hemoglobin, $12.5 \mathrm{~g} /$ $\mathrm{dl}$; and platelet, $274.000 / \mathrm{mm}^{3}$. ESR was $9 \mathrm{~mm} /$ $\mathrm{hr}$. The C-reactive protein was $<3.14 \mathrm{mg} / \mathrm{L}$. Routine biochemistry was within normal range. Tuberculosis skin (PPD) test was negative. Serum total IgE level was $1.986 \mathrm{IU} / \mathrm{ml}$. Pulmonary function tests in our outpatient clinic revealed moderate obstructive pattern with positive reversibility (forced vital capacity [FVC]: 52\%, 71\%; forced expiratory volume in 1 second [FEV1]: 63\%, 83\%; FEV1/FVC: $115 \%, 114 \%$ (pre- and post-bronchodilation values; respectively). According to modified Rosenberg-Patterson criteria, ${ }^{14}$ diagnosis of ABPA was made with positive 7 out of 8 major criteria (Table I). Those 7 major criteria met by the patient were perihilar opacities, central bronchiectasis, eosinophilia, high total IgE, specific IgE $(38.6 \mathrm{KU} / \mathrm{L})$ and IgG antibodies $(135 \mathrm{mg} / \mathrm{L})$ to $\mathrm{AF}$, twice positive skin prick test for AF (wheal of $4 \times 4 \mathrm{~mm}$ ) and reduced FEV1/ FVC on spirometry. Galactomannan antigen was found to be negative. Sputum culture, PCR, T-spot ${ }^{\circledR}$ and Quantiferon ${ }^{\circledR}$ tests for Mycoplasma tuberculosis were negative. Sweat test for CF was negative twice in our and another institution. The entire coding regions of 27 exons of the cystic fibrosis transmembrane conductance regulator gene (CFTR) were amplified by polymerase chain reaction (PCR) using specific primers and the DNA sequencing results were normal. Bronchoscopy showed mucus plugging in the airways and bronchoalveolar lavage (BAL) fluid examined. Cytology of the BAL mostly showed leukocytosis (62\% neutrophilic granulocytes) and gram negative

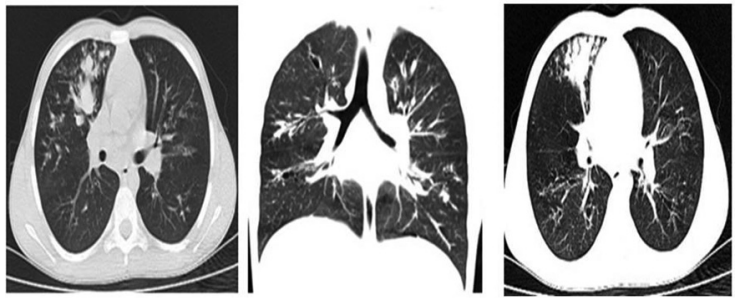

Fig. 2a-c. Before treatment; computed tomography scan of chest shows dilatation and bronchial wall thickening with central bronchiectasis in right upper lobe anterior and posterior segments and left lower lobes antero-basal segments, ground glass and tree in bud appearances.
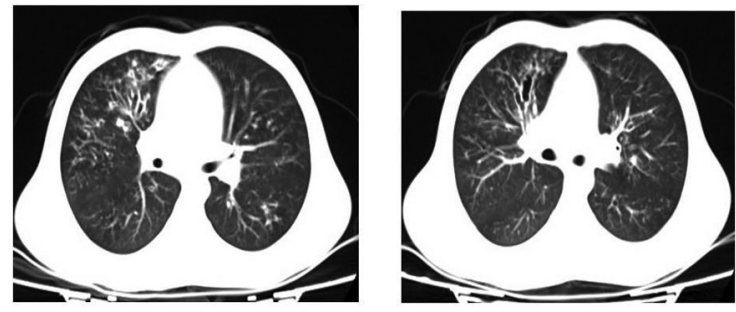

Fig. 2d,e. Lung CT shows decrease in bronchiectasis, ground glass and tree in bud appearances in our patient after 2 months of combined treatment.

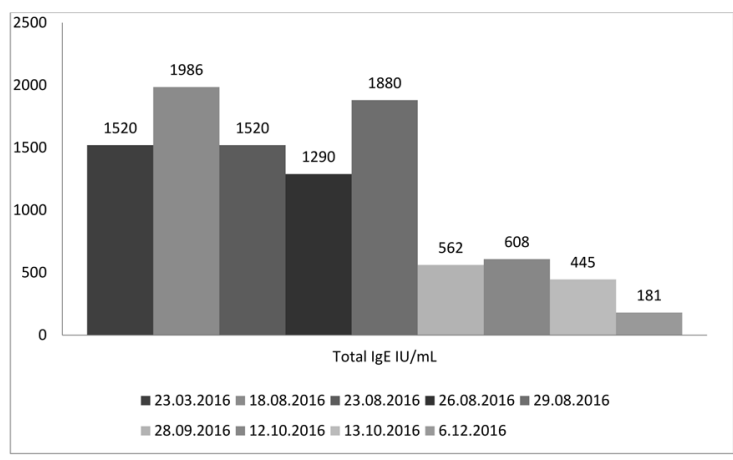

Fig. 3. Total IgE levels $(\mathrm{IU} / \mathrm{mL})$ of our patient over 9 months time.

bacillus. Pseudomonas aeruginosa was isolated from the BAL fluid culture and ceftazidime treatment ( $150 \mathrm{mg} / \mathrm{kg} /$ day) was given for 14 days. ANA and ANCA test results were negative. Serum humoral antibody levels (IgG, IgA, IgM and IgG1- IgG2- IgG3- IgG4) were normal. T-cell subtypes were also detected to be within normal limits.

Intravenous prednisolone ( $1 \mathrm{mg} / \mathrm{kg} /$ day, $30 \mathrm{mg} /$ day for 2 weeks) and oral itraconazole (200 $\mathrm{mg} /$ day, 4 months) treatments were started. After 14 days of oral prednisolone $(1 \mathrm{mg} / \mathrm{kg} /$ day) continued on alternate day for 2 months, it was tapered $4 \mathrm{mg}$ down every other week for 3 months and stopped at 4th months. After 
4 months, oral itraconazole reduced to 100 $\mathrm{mg}$ /day and continued for another 4 months and discontinued at the end of 8th month. During steroid therapy his plasma glucose levels increased and remained high at the $6^{\text {th }}$ week of treatment. All hormonal evaluations including insulin, C-peptid, cortizole, prolactin, tiroid hormones, amylase and lipase were found to be normal. Diagnosis of steroid-induced diabetes mellitus was made by our endocrinologist and insulin treatment had to start. After tapering steroid dose down, his blood glucose levels decreased and remained within normal ranges. In addition to high glucose levels, mildmoderate hirsutism developed. After 14 days of admission, he was discharged. During follow-up his asthma has remained under control with the maintenance therapy of montelukast $(5 \mathrm{mg}$, qd) and budesonid $80 \mu \mathrm{g}+$ formoterol fumarat dihidrat $4.5 \mu \mathrm{g}$ (bid). Under this treatment, his symptoms resolved day by day. At followup, control chest radiograph (Fig. 1b) showed improvement on bilateral perihilar opacities and central bronchiectatic lesions seemed to resolve on control CT (Fig. 2d,e). During and after the treatment, patient's total serum IgE decreased gradually (Fig. 3). Informed consent was obtained from patient's legal guardian (parents).

\section{Discussion}

The clinical presentation of ABPA varies from mild bronchospasm (asthma) to fibrotic parenchymal lung disease. ${ }^{14,15}$ Although very few authors have reported ABPA development in pediatric asthma patients, it could be suspected in uncontrolled asthma that might be related with fungal sensitization. If undetected or inadequately treated, ABPA causes destruction in respiratory tract, bronchiectasis, and/or lung fibrosis, consequently considerable morbidity and mortality. ${ }^{3,10}$ Our patient's worsening clinical symptoms and pulmonary functions suggesting uncontrolled asthma was detected to be associated with bronchiectasis induced by ABPA.

The definite diagnosis of ABPA is not simple due to overlapping clinical and radiological signs. ABPA diagnosis is made by a group of clinical, laboratory, and radiographic findings including asthma, elevated total IgE level, blood eosinophilia, evidence of sensitization to AF, recurrent pulmonary parenchymal infiltrates and bronchiectasis. ${ }^{16}$ The classic RosenbergPatterson criteria are most often utilized for the ABPA diagnosis in non-CF patients. ${ }^{14}$ The existence of six out of the eight major criteria makes the diagnosis almost certain. In a 2012 review, ${ }^{1}$ the minimal criteria necessary for the diagnosis of ABPA were defined as: (1) asthma or CF with worsening of lung function, (2) skin prick test positivity to Aspergillus species, (3) total serum IgE level of $1.000 \mathrm{ng} / \mathrm{ml}$ (416 $\mathrm{IU} / \mathrm{ml}$ ) or higher, (4) amplified Aspergillus species-specific IgE and IgG antibodies, and (5) chest radiographic infiltrates. Additional criteria consist of peripheral blood eosinophilia, Aspergillus species serum precipitating antibodies, central bronchiectasis, and Aspergillus species-containing mucus plugs. ${ }^{5}$ Our patient fulfilled $7 / 8$ classic major criteria for ABPA diagnosis (Table I). In the absence of typical proximal (central) bronchiectasis, the disorder is called seropositive ABPA (ABPA-S) in place of ABPA with central bronchiectasis (ABPA-CB). 5,7,14 Our patient had ABPA-CB.

Although ABPA is known to occur mostly with underlying asthma and $\mathrm{CF},{ }^{16,17}$ there have been several published reports on ABPA development without previously diagnosed underlying disease such as asthma. ${ }^{18}$ Although our patient was also treated for pneumonia several times after being diagnosed and treated for asthma, on the other hand, clinical and CXR findings suggested tuberculosis infection in our patient. Tuberculosis is somewhat of a concern in Turkey; therefore the patient was admitted for tuberculosis work-up and further investigation. Tuberculosis should be considered in the differential diagnosis of ABPA. ${ }^{19}$ This is apparent in a study, as $38.4 \%$ of ABPA patients had a record of anti-tuberculosis treatment. ${ }^{20}$ However, tuberculosis was ruled out by radiologic and laboratory evaluations in our patient. With the clinical and laboratory findings, our patient was considered to have uncontrolled asthma. Key point in the diagnosis of ABPA is suspicion and ABPA should be considered in patients with uncontrolled asthma symptoms. During further investigation, his spirometry showed moderate obstruction with positive reversibility and then the diagnosis of asthma was confirmed. Like our case, diagnosis of ABPA might be delayed because of clinical signs and symptoms mimicking recurrent pneumonia or tuberculosis. In our 
search of the literature from Turkey, we have come across just one case report of 3 adult asthmatic ABPA cases by Erkekol et al. ${ }^{21}$ To the best of our knowledge, this will be the first reported case of ABPA with underlying pediatric asthma from Turkey.

CFTR gene mutations have been reported to be increased in asthmatic patients who developed ABPA. In a meta-analysis, Agarwal et al. ${ }^{22}$ also demonstrated a higher incidence of heterozygous CFTR mutations in asthmatic ABPA patients. However, in our patient DNA sequence analysis for CFTR mutations was normal. In the literature, early colonization of the respiratory tract with Pseudomonas aeruginosa was considered to be related with the development of ABPA in younger patients. ${ }^{4}$ In our patient Pseudomonas aeruginosa was isolated from the BAL fluid culture and we were not sure whether this colonization was associated with the development of ABPA or not. Patterson et al. ${ }^{23}$ categorized ABPA into 5 stages dependent on asthma severity and radiographic or laboratory findings. In this classification, stage I is the early acute phase of ABPA. In stage II, the disease in remission; the infiltrates clear, symptoms decrease and the serum IgE value reduces but usually remains elevated. It looks like our patient is currently at stage II.

Some authors presume that the ABPA is missed in numerous cases with reports of mean diagnostic latency of even 10 years between the beginning of symptoms and the diagnosis. ${ }^{24}$ In our case, the delay between the onset of symptoms and the diagnosis was just a couple of years. There are 2 essential causes for the delay in ABPA diagnosis: the disorder seems to be uncommon and unspecific symptoms can be simply misunderstood as exacerbations of an underlying disease.

ABPA should be suspected in an asthmatic patient if they have the following: (1) new or recent infiltrate(s) implying pneumonia or tuberculosis on lung radiography or thorax CT that have not cured with antibiotics and standard physiotherapy, (2) clinical and laboratory findings suggesting uncontrolled asthma despite adequate therapy, (3) unresolved pneumonia and bronchiectasis irrespective of history of asthma. ${ }^{19}$ Moreover, in the differential diagnosis of ABPA, pulmonary exacerbations by bacterial or viral agents; CF, asthma; atopy; severe small airways disease; gastroesophageal reflux aspiration; pneumothorax; hyperimmunoglobulin E and Churg-Strauss syndrome should be ruled out. Since our patient fulfilled 7/8 diagnostic major criteria suggested by Rosenberg-Patterson, ${ }^{14}$ the other conditions were easily ruled out.

Management of ABPA is based on clinical symptoms. Main goals of treatment are reduction of pulmonary inflammation, prevention of progression of the disease and controlling exacerbations. ${ }^{16}$ Treatment essentially consists of systemic corticosteroid with/without itraconazole. ${ }^{25}$ Oral corticosteroids are the therapy of choice for ABPA. Oral itraconazole treatment is still controversial. Antifungal agents like itraconazole are generally used in CF-ABPA patients; it is thought to diminish the fungal load of the respiratory tract. ${ }^{26}$ There is some evidence that itraconazole has a steroid sparing effect and potentially prevent the recurrence of ABPA. ${ }^{27}$ A recently reported clinical study showed that even refractory pediatric asthma and children sensitized to fungal antigen might benefit from adjunct treatment with itraconazole. Some of the published reports also have shown beneficial effects of combination treatment of itraconazole with prednisolone daily compared with only prednisolone in terms of controlling exacerbations, reduced sputum production, reduced total IgE, better remission of radiological findings and improved FEV1.28

Follow-up of the patient could be done by observing clinical symptoms and total IgE levels. Reducing total IgE levels $35-50 \%$ of the value at the time of diagnosis is expected after starting treatment. ${ }^{26}$ In addition, these patients should be evaluated for adverse effects of long term systemic steroid and itraconazole use. Our case has been successfully treated with combination treatment of methylprednisolone and itraconazole. Clinical symptoms and radiological findings improved, and also total IgE levels decreased. Although these were satisfactory results, he experienced uncontrolled high blood glucose levels caused by steroidinduced diabetes mellitus as a side effect. Fortunately, besides using insulin, tapering the steroid dose down and eventually stopping the steroid brought his blood glucose down to a normal level. 
In conclusion; although it is a rare disease, the physicians should keep ABPA in mind in pediatric patients presenting with uncontrolled asthma despite adequate therapy, unresolved recurrent pneumonia and in those complaining of intractable cough. Also evaluating serum total IgE levels and peripheral eosinophilia with simple skin prick test in a suspected child is easy and rapid way of diagnosis. Even if a patient has no clear-cut history of asthma or central bronchiectasis on lung CT, physicians should be vigilant for potential ABPA-S disorder when radiologic consolidations do not get better with correct antibiotics, if serum total IgE and blood eosinophil levels are high. ${ }^{5}$ Early and correct ABPA diagnosis and aggressive treatment can diminish needless antibiotic treatments and prevent development of irreversible fibrotic lung damage.

\section{REFERENCES}

1. Knutsen AP, Bush RK, Demain JG, et al. Fungi and allergic lower respiratory tract diseases. J Allergy Clin Immunol 2012; 129: 280-291; quiz 292-293.

2. Hinson KF, Moon AJ, Plummer NS. Broncho-pulmonary aspergillosis; a review and a report of eight new cases. Thorax 1952; 7: 317-333.

3. Knutsen AP. Allergic bronchopulmonary aspergillosis in asthma. Expert Rev Clin Immunol 2017; 13: 11-14.

4. Mussaffi H, Greif J, Kornreich L, et al. Severe allergic bronchopulmonary aspergillosis in an infant with cystic fibrosis and her asthmatic father. Pediatr Pulmonol 2000; 29: 155-159.

5. Shin JE, Shim JW, Kim DS, Jung HL, Park MS, Shim JY. Case of seropositive allergic bronchopulmonary aspergillosis in a 10-year-old girl without previously documented asthma. Korean J Pediatr 2015; 58: 190193.

6. Kumar R, Gaur SN. Prevalence of allergic bronchopulmonary aspergillosis in patients with bronchial asthma. Asian Pac J Allergy Immunol 2000; 18: 181-185.

7. Singh M, Das S, Chauhan A, et al. The diagnostic criteria for allergic bronchopulmonary aspergillosis in children with poorly controlled asthma need to be re-evaluated. Acta Pædiatrica 2015; 104, e206-e209.

8. Chetty A, Bhargava S, Jain RK. Allergic bronchopulmonary aspergillosis in Indian children with bronchial asthma. Ann Allergy 1985; 54: 46-49.

9. Eaton T, Garrett J, Milne D, Frankel A, Wells AU. Allergic bronchopulmonary aspergillosis in the asthma clinic. A prospective evaluation of CT in the diagnostic algorithm. Chest 2000; 118: 66-72.

10. Denning DW, Pleuvry A, Cole DC. Global burden of allergic bronchopulmonary aspergillosis with asthma and its complication chronic pulmonary aspergillosis in adults. Med Mycol 2013; 51: 361-370.
11. Wang JL, Patterson R, Rosenberg M, Roberts M, Cooper BJ. Serum IgE and IgG antibody activity against Aspergillus fumigatus as a diagnostic aid in allergic bronchopulmonary aspergillosis. Am Rev Respir Dis 1978; 117: 917-927.

12. Chauhan B, Santiago L, Hutcheson PS, et al. Evidence for the involvement of two different MHC class II regions in susceptibility or protection in allergic bronchopulmonary aspergillosis. J Allergy Clin Immunol 2000; 106: 723-729.

13. D'Urzo AD, McIvor AR. Case report: allergic bronchopulmonary aspergillosis in asthma. Can Fam Physician 2000; 46: 882-884.

14. Rosenberg M, Patterson R, Mintzer R, Cooper BJ, Roberts M, Harris KE. Clinical and immunologic criteria for the diagnosis of allergic bronchopulmonary aspergillosis. Ann Intern Med 1977; 86: 405-414.

15. Greenberger PA, Patterson R. Allergic bronchopulmonary aspergillosis and the evaluation of the patient with asthma. J Allergy Clin Immunol 1988; 81: 646-650.

16. de Almeida MB, Bussamra MH, Rodrigues JC. Allergic bronchopulmonary aspergillosis in paediatric cystic fibrosis patients. Paediatr Respir Rev 2006; 7: 67-72.

17. Ionescu MD, Balgradean M, Marcu V. Allergic bronchopulmonary aspergillosis in patient with cystic fibrosis - a case report. Maedica (Buchar) 2014; 9: 387-390.

18. De H, Azad SM, Giri PP, Pal P, Ghosh A, Maitra A Two cases of non-cystic fibrosis (CF) bronchiectasis with allergic bronchopulmonary aspergillosis. Respir Med Case Rep 2017; 20: 68-71.

19. Kumar R. Mild, moderate, and severe forms of allergic bronchopulmonary aspergillosis: A clinical and serologic evaluation. Chest 2003; 124: 890-892.

20. Kumar R, Goel N. Allergic bronchopulmonary aspergillosis: A clinico-serological correlation with radiologic profile. J Asthma 2013; 50: 759-763.

21. Öner Erkekol F, Bavbek S, Göksel Ö ve ark. Allerjik bronkopulmoner aspergilloz: 3 olgu nedeniyle. Tüberküloz ve Toraks Dergisi 2007; 55: 418-428.

22. Agarwal R, Khan A, Aggarwal AN, Gupta D. Link between CFTR mutations and ABPA: A systematic review and meta-analysis. Mycoses 2012; 55: 357-365.

23. Patterson R, Greenberger PA, Radin RC, Roberts M. Allergic bronchopulmonary aspergillosis: Staging as an aid to management. Ann Intern Med 1982; 96: 286-291.

24. Kirsten D, Nowak D, Rabe KF, Mgnussen H. Diagnosis of bronchopulmonary aspergillosis is often made too late. Med Klin (Munich) 1993; 88: 353-356.

25. Greenberger PA. Clinical aspects of allergic bronchopulmonary aspergillosis. Front Biosci 2003; 8: s119-s127.

26. Santos A, Loureiro G, Faria E, Chieira C. Itraconazole, an effective adjunctive treatment for allergic bronchopulmonary aspergillosis. J Investig Allergol Clin Immunol 2009; 19: 404-408. 
27. Agarwal R. Allergic bronchopulmonary aspergillosis. Chest 2009; 135: 805-826.

28. Davidsen JR, Madsen PH, Laursen CB. Uncontrolled asthma and recurring pulmonary opacities: Just asthma? BMJ Case Rep 2014; 2014. 